\title{
Evaluation of Art Students' Academic Performance in English Language Learning
}

\author{
Li Fengxia \\ Department of Common Courses, Shandong University of Art and Design, Jinan, Shandong, China (lifx0202@ 126.com)
}

\begin{abstract}
This paper introduces the strategies of evaluating the art students' academic performance in English learning in an art college and analyzes the significance of multi-evaluation in the academic performance of college students. This paper also focuses upon the existing problems in evaluation and explores the effective ways in promoting English language teaching and learning.
\end{abstract}

Key words — evaluation, academic performance, art students

\section{对艺术类大学生的英语学习状况的评价 \\ 李凤霞 \\ 山东工艺美术学院公共课教学部, 济南, 山东, 中国}

\begin{abstract}
摘 要 本文概述了在大学英语教学中针对艺术类大学生的英语学习的评价策略以及多元化评价的重要性, 分析了在评价实施的 过程中存在的问题并探索如何通过有效的评价体系促进英语教学质量的提高。
\end{abstract}

关键词 评价, 学习表现, 艺术生

1. 引言

在语言学习中, 评估指对学生个体语言的学习过程、 结果、能力等方面的数据收集, 包括需求分析、水平的测 量、课程目标的达成信息, 以及任务操作与完成信息 [1]。 评估是通过多种渠道收集有关学生学习过程以及语言能 力、态度、参与、认知发展等方面的信息, 借以促进学习 和教学[2]。而评价是对所评估信息进行解释和判断的过程, 是在评估的基础上分析评估数据对教学和学习的总体的价 值作用。评估过程是评价过程的一部分, 是评价的基础。 评价与评估是一个交互进行的过程。2007 年教育部高教司 颁发的《大学英语课程要求》明确指出“教学评估是大学英 语课程教学的一个重要环节。全面、客观、科学、准确的 评估体系对于实践教学目标至关重要”[3]。

随着建构主义学习理论和价值论的发展, 作为学习者 的学生在评价中的主体地位得到越来越多的认可。建构主 义基于对认识论的理解, 认为学习过程是学习者积极主动 地建构自己的认识过程。建构主义强调在教学过程中, 要 激发学生的主体活动, 强调合作解决问题; 在评价中强调 评价者与被评价者通过协商, 共同完成心理的建构。学生 在评价中不仅是评价的客体, 同时处于主体的位置。研究
者们对形成性评价方面的研究最为普遍。形成性评价作为 教学和学习的有机组成部分, 是一种在教学和学习过程中 对信息的收集、整理和解释的过程[4]。形成性评价作为教 学和学习的一部分在教学和学习的过程中发生, 运用各种 情景下学生的表现信息作为评价的依据。而评价以反馈信 息、指导学生和促进学习为目的, 学生作为主体参与到评 价的过程之中, 其主体性和创造性受到尊重。课堂教学的 主要目标是帮助学生达到一系列既定的学习目标, 包括学 生在智力、情感和生理方面的积极变化。所以课堂评价的 重点放在如何使评价更好地达到为学生进步提供有用的反 馈信息的目的, 评价必须与语言学习的各发展阶段相结合, 评价数据应用于调整教学策略和改善学生的学习方式等方 面。

\section{2. 评价的开展}

该研究基于一所艺术高校的大学英语教学情境, 研究 对象是大学一年级和大学二年级的学生。该校实行学分制, 大学英语分为四个学期开设。学校的评价方式是传统的期 末考试方式, 自 2011 年开始实行期末与平时成绩相结合的 考评方法, 目的是为了增强考评方式的科学性和合理性, 
通过对学生的综合学习情况进行多元化的评价, 进而达到 提高大学英语教学效果的目标。学生的期末考试包括笔试 和机考两种方式, 笔试部分是主观题, 机考部分是客观题, 成绩占总成绩的百分之七十。平时成绩占总成绩的百分之 三十, 平时成绩主要是由任课教师根据课堂教学实际情况 进行评定的, 评定的内容包括不定期考勤、课堂问答、对 话练习、课堂讨论、课堂测试以及课后作业等几个方面。 在英语教学过程中, 教师鼓励学生们进行英语能力自评和 互评活动, 采用的工具是教育部颁布的的《大学英语课程 要求》里推荐使用的《学生英语能力自评/互评表》。学生 在自评和互评时的各项微技能以 《大学英语课程要求》里 规定的一般要求的内容为标准参照。因为该校是一所设计 类艺术高校, 根据 2009 年、 2010 年和 2011 年连续三年的 本科生入学英语成绩的调查和分析得出结论: 与普通文理 科本科生的高考英语成绩相比, 艺术类本科生的高考英语 成绩普遍较低, 英语学习能力相对较弱, 所以该校的英语 教学目标是完成《大学英语课程要求》的一般要求。

在学生自评和互评方面, 教师们在教学活动开始时, 向学生们介绍本学期的英语教学目标和教学要求, 帮助他 们详细了解自评/互评表中列举的各项微技能。然后在教学 进行的过程中, 教师们定期要求学生们进行自评和互评。 通过自评和互评, 帮助学生们能了解自己对语言技能的掌 握情况, 及时发现学习中的问题, 根据实际情况调整学习 策略和学习行为。

\section{3. 分析和讨论}

\section{1 评价的主体}

该校学生的平时成绩占总成绩的百分之三十, 平时成 绩的评定基本上是由任课教师来完成的。任课教师通过观 察和了解每个学生的学习行为和课堂表现, 以及通过一些 课堂测试、课堂问答等方式作出初步的判断, 记录下详细 的考核数据, 主要以发现问题、梳理指导、促进发展为目 的, 但也为对学生划分等级和甄别提供支持。当教师实施 评价时, 所评价的是学生的学习行为是否促进了自身的发 展, 学生通过学习是否达成了学习目标。学生与学习行为 之间构成一种价值关系, 教师是作为评价的主体判断学生 及其行为之间的价值关系。学习意味着学生对知识的扩充 和对相关技能的习得。王笃勤认为在学习过程中, 学生以 自己的主观能动性把新学的知识纳入自己的已有图式, 建 构着自己的意义世界。学生是学习和认知的主体, 在评价 中是价值的主体。如果评价由学生来实施, 学生就承担了 双重角色, 既是评价主体, 又是价值主体。学生所评价的 是学习活动和评价活动与自己的价值关系。学生在参与自
评和互评活动的过程中, 可以看到自己在学习中的进步, 同时也发现自己在学习过程中存在的问题。在问题解决之 后, 进而确立新的学习目标。互评可以促使学生之间达成 互动, 不仅有利于增加他们对语言形式的注意, 提高他们 对语言准确性的意识, 还有利于营造合作性的学习氛围, 有利于促进学生主动建构自己的知识。其实真正意义上的 学习, 是由学生自我评价的, 因为只有学生最清楚某种学 习是否满足自己的需要, 是否有助于让他们获得想要知道 的东西。学生对自我的评价、判断, 也是一种学习手段和 一个体验过程。学生们参与评价的过程, 其实也是一种学 习的过程。学生可以在评价中对自己的学习行为进行反思、 有所领悟, 从而进一步发现自我、认识自我和提升自我。 总之, 让学生参与到评价活动中来, 不仅能增强他们的学 习自主性和能动性, 还有利于评和学的融合, 因为评价活 动本身也可作为学习活动的一部分。

\section{2 评价的影响}

在教与学的过程中, 评价担当着重要的角色。评价提 供的数据, 不但会影响教师对教学方法、教学材料和教学 方向的决定, 也会影响学生在学习上的决定。在语言测试 中, 把测试对教学的影响一般称为“回波效应”[5]。在形成 性评价过程中, 正面回波效应应包括两个含义: 首先是对 教师的影响。教师在评价过程中发现问题, 因而制定新的 教学计划, 在下一步教学中加以改进。另外是评价对学生 的影响。“如果我们把辩论、讨论、论文和问题解决纳入测 试系统, 学生自然就会训练这些活动”[6]。人们的价值取向 的差异, 会导致人们的思维方式和行为方式的不同。所以 评价会对学生的学习方式带来不同程度的影响。如果评价 内容偏重于书本知识, 学生就会倾向于采取接受学习方法; 如果评价内容偏重于创新能力, 学生就会采取探究学习方 式[7]。由此可见, 评价的结果对教和学的各方面都会产生 影响, 这就要求教师必须深入了解每项评价的内容和具体 的目标, 灵活得运用通过评价所获得的资料, 找出学生在 学习中的问题和困难, 及时向他们提供回馈, 并设计切合 学生需要和能力的教学活动, 根据实际情况调整教学计划, 实现以评价来促进教学效果的提高的目标。

\section{3 评价的标准}

教学评价的关键在于教学评价标准的选择。在教学评 价的过程中, 必须做到评价标准与课程标准和教学标准的 一致。标准参照被认为是检验学生外语水平和课程目标的 适当方法[8]。标准参照是根据既定的统一标准来评价学生 的学习行为, 而不是比较参照其他学生的数据, 所以它可 以反映学生是否达到标准, 而不是看学生之间的比较, 这 
样的评价结果是比较客观的、公正的, 也有利于学生根据 自己的具体情况确定学习深度和进度, 进行个性化的学习。

\section{4. 存在的问题}

教学评价结果的信度, 即教学评价的可靠性, 是衡量 教学评价质量的指标之一。如果要提高教学评价结果的信 度, 就要减小误差。教学评价误差的主要来源包括教学评 价量表结构、教学评价主体的松紧度以及教学评价行为目 标难度等等, 这些都有可能成为教学评价的干扰因素。评 价误差有时来源于对标准理解的不一致性。所以评价主体 能否一致地理解和掌握评分标准, 是提高评分信度的关键 [9]。教师在评价时如果能够区别学生不同的行为和表现水 平的能力, 始终如一地把握评分松紧度, 就能有效地控制 由评分教师引起的评分误差, 那么, 由评分教师们自身变 化引起的评分误差就比较小; 反之, 如果教师的评分要求 忽紧忽松, 就无法保证评分的稳定性。如果教师之间的评 价的松紧度差异得到控制, 那么评价结果的信度将会较高。 在学生互评时, 如果评价主体不能对学生显著不同的独立 的行为特征作出区分, 就有可能出现评价模糊现象; 或者 评价数据聚集在评分量表的中间等级附近, 出现评分趋中 现象[10]。

\section{5. 结语}

当前的教育目标的定位是培养创新型人才, 为社会的 发展和学生的发展及学生的终身学习打下良好的基础。学 生是学习的主体, 不论年龄、知识、能力如何, 学生的学 习应当向着个性化和自主学习的方向发展。学生在学习的 过程中应具有主体性, 即主动性、创造性和独特性。教师 在课堂教学中的进行评价时, 既要关注评价过程, 又要关 注评价方法。教师不仅要引导学生们从评价的过程中发现 学习中存在的问题, 并找到的不同方法去解决问题, 还要 引导学生对评价结果进行有效的交流和总结, 把自己的所 思所想和所感所悟都一一梳理出来, 与大家共同分享。教 学中的评价具有阶段性, 也具有延伸性, 所以学习的过程
性、体验性和发展性都是不容忽视的。教师们要充分发挥 课堂评价的教育和发展功能, 使之真正对教和学起到促进 作用。教师们还应不断地改进对学生们的评价方式和方法, 倡导学生进行自评和互评, 力求使评价标准、评价内容和 评价方法向多元化发展。只有这样, 才能达到以评促学的 目标, 才能有效提高教学效果和教学质量, 从而实现人才 培养目标。

\section{参考文献(References)}

[1] Lynch,B.K. Language and program evaluation. Edinburgh: Edinburgh University Press Ltd, 2003:1.

[2] Duqin, Wang. Authentic Assessment. Beijing: Foreign language Teaching and Research Press, 2007.

[3] Higher Education Department of Education Ministry. College English Curriculum Requirements. Beijing: Higher Education Press, 2007.

[4] Bryant, S.L., L.T.S.John, A.A.Timmins \& J.A.H.Williams. Formative assessment for enhancing learning: An annotative bibliography. Hong Kong: Hong Kong Institute of Education, 2002.

[5] Zunmin,Wu. The Theory and Practice of English Language Testing. Beijing: Foreign language Teaching and Research Press, 2002.

[6] Resnick,L.B. \& D.P.Resmck.Assessing the thinking curriculum: new tools for educational reform. In: B.R. Gifford and M.C.O'Connor. (eds.)Changing Assessment: Alternative Views of Aptitude, Achievement and Instruction. Boston: Kluwer Academic Publishers, 1992: 59.

[7] Xianhua,Zhang. The Revolution of Education Thoughts. Beijing: Peking University Press, 2005.

[8] Jimei,Xia.Foreign Language Course Design: Modern Theory and Practice. Shanghai: Shanghai Foreign Language Education Press, 2003.

[9] Juan,Luo. Analysis of English Writing Assessment. Foreign Languages in China,2008 (5):61-66.

[10] Qun,Zhou. Study on the Effect of Subjective Evaluation. Testing Research, 2007(3): 37-47. 